\title{
RESONANCE TRAPPING OF POSITRONS IN SOLIDS
}

\author{
J. DRYZEK \\ Institute of Nuclear Physics, Radzikowskiego 152, 31-342 Kraków, Poland
}

Basing on a new approach to the calculations of the positron trapping rate, an attempt 'was made to explain the prevacancy effects observed in positron annihilation measurements in metals. The defect in the calculation is represented by a complex potential: the real part of it is responsible for the scattering and the imaginary part - for the trapping of incoming positrons. It is shown that the resonance trapping of the positron $s$-wave and $p$-wave at low temperature and $d$-wave and $f$-wave in prethermal regions provide substantial contributions to the trapping rate of positrons. The calculations were done for the point defect and for the dislocation, taking into account the thermal distribution of the positron energy and the thermal expansion of the crystal lattice. The resonance trapping of positrons could be responsible for prevacancy effects in metals and could help to solve some problems in the study of positron annihilation.

PACS numbers: 78.70.Bj

Positron trapping by a defect was investigated as a scattering of a positron at the complex spherically symmetric potential which represents that defect

$$
V(r)= \begin{cases}-V_{0}(1+\mathrm{i} \xi) & \text { for }|\boldsymbol{r}|<R, \\ 0 & \text { for }|\boldsymbol{r}|>R,\end{cases}
$$

where $V_{0}$ describes the average potential energy felt by the positron within the defect, $R$ is the defect radius, $\xi$ is the absorption coefficient and $\mathrm{i}=\sqrt{-1}$.

The real part of this potential is responsible for the elastic scattering and the imaginary part - for the trapping of the incoming positron [1, 2]. follows:

The asymptotic behaviour of the positron wave function can be written as

$$
\psi(r)=\phi_{\text {in }}(r)+\psi_{\text {out }}(r)=\mathrm{e}^{\mathrm{i} k z}+\frac{\mathrm{e}^{\mathrm{i} k \cdot r}}{r} f(\theta)+\mathrm{O}\left(\frac{1}{r^{3}}\right),
$$

where $f(\theta)$ is called the scattering amplitude.

The solution of the Schrödinger equation with the potential (1) and positive energy make it possible to find $\psi(r)$ and hence the scattering amplitude which can be expressed by the relation

$$
f(\theta)=\frac{1}{k} \sum_{l=0}(2 l+1) \mathrm{e}^{\mathrm{i} \delta_{l}} \sin \left(\delta_{l}\right) P_{l}(\cos (\theta)),
$$


where $\delta_{l}$ is the phase shift between ingoing and outgoing waves. Then the positron trapping rate at the defect, which is represented by the complex potential (1), is the following:

$$
\nu=n \frac{\pi \hbar^{2}}{m^{2} v} \sum_{l=0}(2 l+1)\left(1-\left|\mathrm{e}^{2 \mathrm{i} \delta_{l}}\right|^{2}\right)
$$

where $n$ is the atomic density of the medium, $v$ is the velocity of scattered positron, $\hbar$ is the Planck constant and $m$ is the positron effective mass. The phase shift $\delta_{l}$ is defined by the potential $V(r)$, which can be obtained after some calculations. In our consideration the positron before annihilation has a thermal energy. Hence the positron velocity is

$$
v=\sqrt{\frac{3 k_{\mathrm{B}} T}{m}}
$$

where $k_{\mathrm{B}}$ is the Boltzmann constant. The properties of the defect are represented by two parameters: the first parameter is the absorption coefficient $\xi$ and the second is

$$
X_{0}=\sqrt{\frac{2 m}{\hbar^{2}} V_{0}} R \text {. }
$$

The formalism presented indicates that the positron trapping rate will have characteristic large scale resonances which mainly depend upon the value of parameter $X_{0}$. The amplitude and the width of the resonances depend upon the absorption coefficient $\xi$. In Fig. 1 the trapping rates are presented as a function
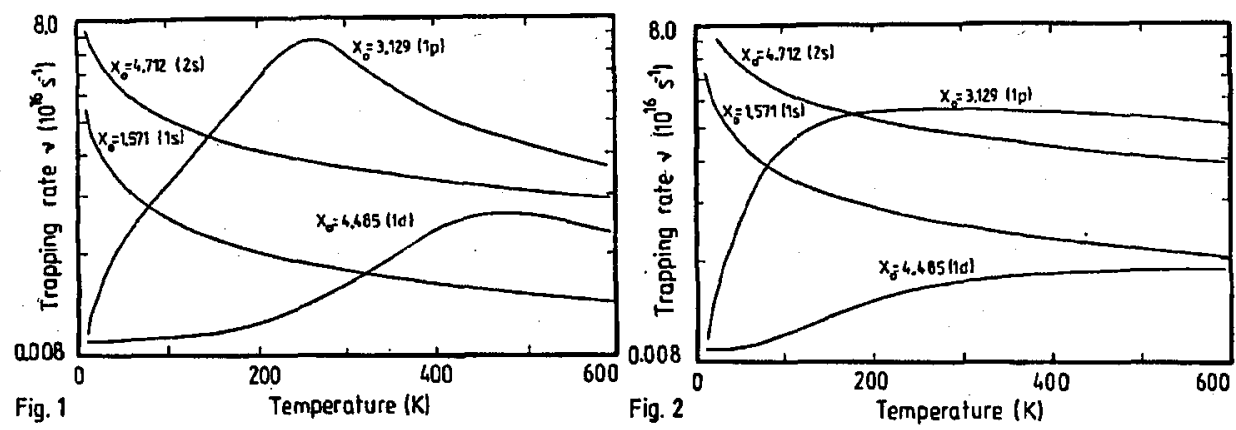

Fig. 1. Total positron trapping rate at the point defect (Eq. (3)) as a function of temperature for different values of $X_{0}$ parameter. The other parameters were following: $R=0.173 \mathrm{~nm}, n=4.64 \times 10^{22}$ atoms $/ \mathrm{cm}^{3}$ (like in Cd), $\xi=0.001$ and $m^{*}=m_{\mathrm{e}}$.

Fig. 2. Tótal positron trapping rate at the point defect as a function of temperature for different values of $X_{0}$ parameter. In the calculations the thermal distribution of the positron momentum was taken into account according to Eq. (6). The other parameters were: $R=0.173 \mathrm{~nm}, n=4.64 \times 10^{22}$ atoms $/ \mathrm{cm}^{3}, \xi=0.001$ and $m^{*}=m_{\mathrm{e}}$.

of temperature for the resonance of $s-, p$ - and $d$-waves. Thus one can conclude that the resonance of $s-, p$ - and $d$-waves should be present for the thermal energies of a positron and the resonance of $f$-wave for the prethermal energies. In reality, the thermalised positron at temperature $T$ is near the bottom of the positron 

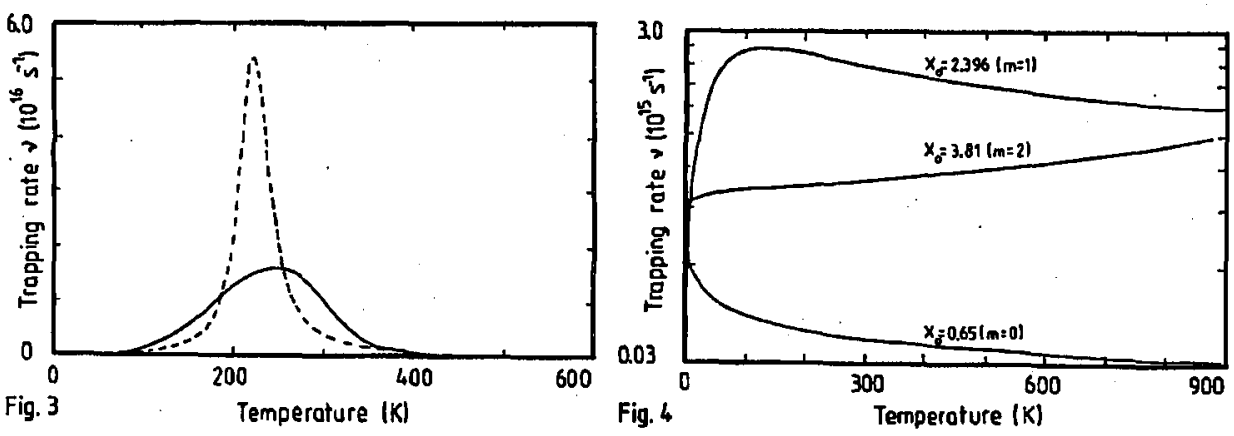

Fig. 3. Total positron trapping rate at the point defect as a function of temperature. Broken curve - the results according to Eq. (3), the thermal expansion of the crystal lattice was assumed $\left(X_{0}(T)=X_{0}(1+\alpha T)\right.$, where $X_{0}=3.11$ and $\left.\alpha=3 \times 10^{-5} 1 / \mathrm{K}\right)$. Full curve - the same calculation but the thermal distribution of positron momentum was taken into account. The other parameters were: $R=0.173 \mathrm{~nm}, n=4.64 \times 10^{22}$ atoms $/ \mathrm{cm}^{3}, \xi=0.001$ and $m^{*}=m_{\mathrm{e}}$.

Fig. 4. Total trapping rate at the dislocation as a function of temperature for different values of $X_{0}$ parameter. The other parameters were: $R=0.184 \mathrm{~nm}, \xi=0.01, m^{*}=$ $1.12 m_{\mathrm{e}}, n=1.29 \times 10^{15}$ atoms $/ \mathrm{cm}^{2}$.

conduction band and its momentum is determined by the Boltzmann distribution. Therefore, the trapping rate, a function of the temperature, for the real medium should be convoluted with the Boltzmann distribution

$$
\nu(T)=\frac{n \pi \hbar^{2}}{m^{2}} \int_{0}^{\infty} \mathrm{d} v \frac{f(T, v)}{v} \sum_{l=0}(2 l+1)\left(1-\left|\mathrm{e}^{2 \mathrm{i} \delta_{l}}\right|^{2}\right),
$$

where

$$
f(T, v)=\left(2 \pi m^{*} k_{\mathrm{B}} T\right)^{-3 / 2} \exp \left(-m^{*} v^{2} / 2 k_{\mathrm{B}} T\right) .
$$

In such a case the resonances should either broaden or vanish and only the resonance of the $s$-wave retains the same shape, see Fig. 2. Temperature also causes the thermal expansion of the crystalline lattice; the radius of the defect $R$ and consequently the $X_{0}$ parameter are the functions of temperature. In first approximation one can assume that the temperature dependence of that parameter is a linear one

$$
X_{0}(T)=X_{0}(1+\alpha T),
$$

where $\alpha$ is the lattice expansion coefficient.

Including of this effect into our calculations gives surprising results. The resonance of $p$-wave which was suppressed by the thermal distribution of positrons motion is now becoming more visible (Fig. 3). Therefore, the temperature is unlikely to destroy the predicted resonances.

Similar calculation can be performed for the trapping of a positron by a dislocation which can be described by a complex potential like (1) taken in two 
dimension. The positron trapping rate in that case is the following:

$$
\nu=n_{\mathrm{p}} \frac{\hbar}{m} \sum_{m=0} \varepsilon_{m}\left(1-\left|\mathrm{e}^{2 \mathrm{i} \delta_{m}}\right|^{2}\right)
$$

where

$$
\epsilon_{m}= \begin{cases}2 & \text { for } m=0 \\ 1 & \text { for } m \neq 0\end{cases}
$$

and $n_{\mathrm{p}}$ is the atomic density on the plane. Trapping by the dislocation also exhibits the resonance properties (Fig. 4).

Figure 5 presents simulation of mean positron lifetime as a function of temperature for Cd assuming that the positron is trapped by the residual defect concentration with the resonance trapping expressed by (6) and (9).

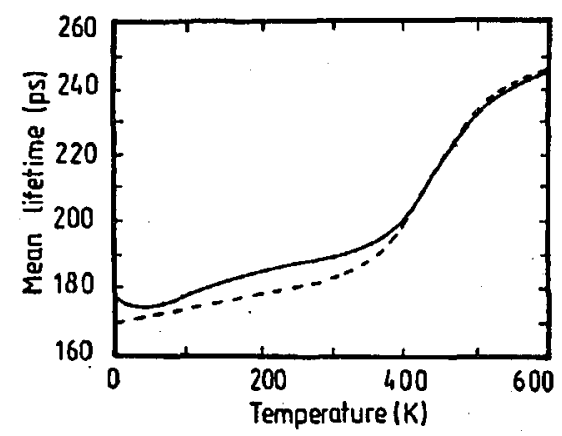

Fig. 5. The plot of positron mean lifetime for $\mathrm{Cd}$ as a function of temperature. Full curve represents the simulation with the residual two types of dislocations with $X_{0}$ parameter equal to $0.64(1 s)$ and $2.365(1 p)$ and the concentration equal to $1.5 \times 10^{-5}$ and $7 \times 10^{-7}$ respectively, at which the resonance trapping took place. The lifetime of positrons in such traps was equal to 230 ps. In the simulation the vacancy formation enthalpy was equal to $H_{f}=0.39 \mathrm{eV}$, the entropy $S_{f}=K_{\mathrm{B}}$ and specific trapping rate at the vacancy thermally generated was equal to $7 \times 10^{13} 1 / \mathrm{s}$. Broken line was obtained neglecting residual vacancies. In both cases we assumed the linear temperature coefficient of $\tau_{\mathrm{f}}$ which was equal to $\alpha=2.5 \times 10^{-4} 1 / \mathrm{K}$.

Although the result of calculations of the trapping rate is somewhat unexpected, it offers a very interesting explanation of the prevacancy effects observed in many metals [3].

\section{References}

[1] H. Feshbach, C.E. Porter, V.F. Weisskopf, Phys. Rev. 96, 448 (1954).

[2] Y. Shirai, J. Takamura, J. Phys., Condens. Matter 1, SA 125 (1989).

[3] L.C. Smedskjaer, in: Positron Solid-State Physics, Ed. W. Brandt, A. Dupasquier, North-Holland, Amsterdam 1983, p. 597. 